Pacific Journal of Mathematics

FINITE-TO-ONE OPEN MAPPINGS ON CIRCULARLY 


\title{
FINITE-TO-ONE OPEN MAPPINGS ON CIRCULARLY CHAINABLE CONTINUA
}

\author{
Philip Bartick ANd Edwin DUda
}

\begin{abstract}
The authors analyze the behavior of a finite-to-one open mapping on a hereditarily decomposable circularly chainable continuum. It is shown that such a mapping behaves similarly to an open mapping on a simple closed curve.
\end{abstract}

0. Introduction. In a previous paper (2), the authors completely described the behavior of a finite-to-one open mapping on a hereditarily decomposable chainable continuum. In this note we will completely describe the behavior of a finite-to-one open mapping on a hereditarily decomposable circularly chainable continuum. This result is a generalization of the following theorem of G. T. Whyburn (8): If $X$ is a simple closed curve and $f(X)=Y$ is a non-constant open mapping onto a Hausdorff space, then $Y$ is either a simple closed curve or an arc. If $Y$ is a simple closed curve, then there is an integer $n$ such that $f$ is topologically equivalent to the mapping $w=z^{n}$ on the unit circle in the complex plane. If $Y$ is a simple arc, then there exists an even integer $k$ such that $f$ is topologically equivalent to the mapping $f(1, x)=\sin (k x / 2)$ for $0 \leq x \leq$ $2 \pi$ from the unit circle $r=1$ in the plane to the interval $[-1,1]$. We use the fundamental results of E. S. Thomas (6) implicitly.

1. Notation and definitions. The word mapping is used to denote a continuous function and a metric continuum $X$ is decomposable if it can be expressed as the union of two proper subcontinua. A continuum $X$ is hereditarily decomposable if every non-degenerate subcontinuum is decomposable. The interior of a subset $D$ is denoted by $\operatorname{int}(D)$ and the closure of $D$ is represented by $\operatorname{cl}(D)$. Two mappings $f(X)=Y$ and $g(Z)=W$ are topologically equivalent if there exist homeomorphisms $h(X)=Z$ and $k(W)=Y$ such that $k g h(x)=f(x)$ for all $x$ in $X$. A mapping $f(X)=Y$ is irreducible if no proper subcontinuum of $X$ maps onto $Y$ under $f$. Suppose $X$ is a continuum which admits a monotone mapping $t$ onto the unit interval such that no point inverse has interior points. A point inverse $t^{-1}(r), r \neq 0$, is called an element of subcontinuity from the left in the upper-semicontinuous decomposition of $X$ induced by $t$ if $t^{-1}(r) \subset \operatorname{cl}\left(t^{-1}[0, r)\right)$. If $r \neq 1$ and $t^{-1}(r) \subset \operatorname{cl}\left(t^{-1}(r, 1]\right)$, then $t^{-1}(r)$ is an element of subcontinuity from the right. The collection of elements of subcontinuity from the left are known to be a dense set in $X(6)$ and the same is true of the elements of subcontinuity from the right. 


\section{Preliminary results.}

LEMMA 1. Let $X$ be a hereditarily decomposable circularly chainable continuum. There is a monotone upper-semicontinuous decomposition $G(X)$ defined on $X$ whose quotient space is a simple closed curve and each element of which has empty interior.

Outline of proof. Let $X=A \cup B$ be a decomposition of $X$ into proper subcontinua. We can assume that $A \cap B$ has no interior points. The continua $A$ and $B$ are chainable and hereditarily decomposable, so that by R. H. Bing's characterization (1) they admit monotone upper-semicontinuous decompositions $G(A)$ and $G(B)$ respectively whose quotient spaces are simple arcs, say $[a, b]$ and $[c, d]$ respectively. Furthermore, no element of $G(A)$ or $G(B)$ has interior points relative to $X$. We can assume that the elements corresponding to $a$ and $c$ meet, as do the elements corresponding to $b$ and $d$. The monotone decomposition $G(X)$ is obtained by taking the union of the elements corresponding to $a$ and $c$ as one element, the union of the elements corresponding to $b$ and $d$ as another element, and the rest of the elements of $G(A)$ and $G(B)$ for the remainder of $G(X)$. This decomposition is upper-semicontinuous, its quotient space is a simple closed curve, and no element of it has interior points.

The following theorem established in (2) is stated in complete detail even though only the first portion is used in this paper.

THEOREM A. Let $f(X)=Y$ be a finite-to-one open mapping, where $X$ is a non-degenerate hereditarily decomposable chainable continuum and $Y$ is a Hausdorff space. Then $X=\cup_{j=1}^{n} K_{j}$, where each $K_{j}$ is a continuum, $f\left(K_{j}\right)=Y$ for each $j, f$ is a homeomorphism on any continuum which is interior to $K_{j}$ relative to $X$, and if $K_{i} \cap K_{j}$ is non-empty for $i \neq j$, then the intersection is contained in a single element $K$ of $G(X)$. If $K$ is an element of subcontinuity from one side, then it is an element of subcontinuity from both sides. If not, then $K$ is the union of two homeomorphic subcontinua which meet in a single element of $G(K), f$ is one-to-one on each of them, and they have the same image. If $f$ is irreducible (i.e., there is only one $K_{j}$ ), then $f$ is a homeomorphism.

LEMma 2. If $f(X)=Y$ is a finite-to-one open mapping, where $X$ is hereditarily decomposable and circularly chainable, and $Y$ is Hausdorff, then $Y$ is hereditarily decomposable and either circularly or linearly chainable. 
Proof. By well-known theorems, $Y$ is a metric continuum. The space $Y$ is hereditarily decomposable, for if $Y$ contained a non-degenerate indecomposable continuum $L$, then by an application of the Brouwer Reduction Theorem there would exist an indecomposable continuum in $X$ which maps onto $L$. The space $Y$ is circularly or linearly chainable by a theorem of E. Duda and J. Kell (3) or by applying a result of W. T. Ingram (5).

\section{Main result.}

THEOREM 1. Let $f(X)=Y$ be a finite-to-one open mapping, where $X$ is hereditarily decomposable and circularly chainable and $Y$ is a Hausdorff space. Then $Y$ is hereditarily decomposable and either linearly or circularly chainable. If $Y$ is circularly chainable, then $f$ is exactly $n$-to-one for some $n$ and a local homeomorphism, and $X=\cup_{j=1}^{n} K_{j}$, where each $K_{j}$ is a continuum, $f\left(K_{j}\right)=Y$ for each $j$, and if $K \subset \operatorname{int}\left(K_{j}\right)$ is a continuum, then $f$ is one-to-one on $K$. If $Y$ is linearly chainable, then there is an even integer $k$ such that $X=\cup_{j=1}^{k} K_{j}$, where each $K_{j}$ is a continuum, $f\left(K_{j}\right)=Y$ for each $j$, and if $K \subset \operatorname{int}\left(K_{j}\right)$ is a continuum, then $f$ is one-to-one on $K$.

Proof. By Lemma 2, $Y$ is a hereditarily decomposable linearly or circularly chainable continuum. By Lemma 1 , there is an upper-semicontinuous decomposition $G(X)$ generating a monotone mapping $g_{1}$ onto a unit circle $S_{1}$, such that each point-inverse of $g_{1}$ is an element of $G(X)$. If $Y$ is circularly chainable, there is a similar upper-semicontinuous decomposition $G(Y)$ generating a monotone map $g_{2}$ onto a unit circle $S_{2}$. If $Y$ is linearly chainable, then by Bing's theorem (1), there is a monotone decomposition $G(Y)$ generating a monotone map $g_{3}$ of $Y$ onto the interval $I=[-1,1]$. Each point-inverse of $g_{1}, g_{2}$, or $g_{3}$, or equivalently, each element of $G(X)$ or $G(Y)$, has empty interior.

It was proved in (2) that if $T \in G(X)$, then $f(T) \in G(Y)$, and if $L \in G(Y)$, then $f^{-1}(L)=\cup_{l=1}^{m} L_{i}$, where $L_{l} \cap L_{j}=\varnothing$ if $i \neq j$ and each $L_{\imath} \in G(X)$.

If $Y$ is circularly chainable, define a mapping $f_{1}$ of $S_{1}$ onto $S_{2}$ by $f_{1}(z)=g_{2} f g_{1}^{-1}(z)$. The mapping $f_{1}$ is well-defined, since $f$ preserves decomposition elements, and $f_{1}$ is continuous, finite-to-one and open, since $f$ has these properties. By the quoted theorem of Whyburn, $f_{1}$ is a local homeomorphism which is topologically equivalent to $z^{n}$ for some integer $n$. If $I_{1}$ is an interval in $S_{1}$ of length less than $2 \pi / n$, then $f_{1} \mid I_{1}$ is a homeomorphism of $I_{1}$ onto $f_{1}\left(I_{1}\right)$. The set $g_{1}^{-1}\left(I_{1}\right)$ is a continuum, and it is a component of $f^{-1} f\left(g_{1}^{-1}\left(I_{1}\right)\right)$, so $f \mid g_{1}^{-1}\left(I_{1}\right)$ is a finite-to-one open mapping. Furthermore, $f \mid g_{1}^{-1}\left(I_{1}\right)$ is irreducible, and hence by Theorem A, it is a homeomorphism. We now know that $f$ is a local homeomorphism and exactly $n$-to-one. 
Let $S_{1}$ be divided into $n$ non-overlapping arcs $I_{j}, j=1, \ldots, n$, each of length $2 \pi / n$, and let $K_{j}=g_{1}^{-1}\left(I_{j}\right)$ for each $j$. Then $X$ is the union of the $K_{j}, f\left(K_{j}\right)=Y$ for each $j$, and if $K \subset \operatorname{int}\left(K_{j}\right)$ then $f \mid K$ is one-to-one.

Suppose $Y$ is linearly chainable. Define the map $f_{2}$ from $S_{1}$ to $I$ by $f_{2}(z)=g_{3} f g_{1}^{-1}(z)$. As before, $f_{2}$ is a continuous finite-to-one open mapping of $S_{1}$ onto $I$, and by Whyburn's theorem, $f_{2}$ is topologically equivalent to the mapping $f(1, x)=\sin (k x / 2)$ for $x$ between 0 and $2 \pi$ and $k$ an even integer. In this case, $S_{1}=\cup_{j=1}^{k} I_{j}$ and $f_{2} \mid I_{j}$ is a homeomorphism of $I_{j}$ onto $I$. Let $K_{j}=g_{1}^{-1}\left(I_{j}\right)$ for each $j$. Then $f\left(K_{j}\right)=Y$ for each $j$, and if $L$ is any continuum in the interior of $K_{j}$, then $f \mid L$ is a finite-to-one open mapping of $L$ onto $f(L)$. Furthermore, it is irreducible, so that $f \mid L$ is a homeomorphism by Theorem A.

The following example illustrates that when $Y$ is linearly chainable, $f \mid K_{j}$ is not necessarily a homeomorphism. Let $X$ be the closure of the graph of $y=\sin (1 / x), x \neq 0, x \in[-1,1]$. Let the endpoints of $X$ be $a$ and $b$. In the unique minimal monotone decomposition $G(X)$ given by Bing's theorem, the only non-degenerate element is the interval from $(0,-1)$ to $(0,1)$ on the $y$-axis. Let $q$ be the quotient map obtained by identifying each point $(x, y)$ in $X$ with $(-x,-y)$. The mapping $q$ is open and exactly 2-to-one except at the origin. Let $Y$ be a disjoint copy of $X$ with endpoints $c$ and $d$ corresponding to $a$ and $b$, respectively. Let $Z$ be the circularly chainable continuum obtained by identifying $c$ with $a$ and $b$ with $d$. Let $p$ be the mapping of $Z$ onto $X$ obtained by folding $Z$. The mapping $f=q \circ p$ is a finite-to-one open mapping of $Z$ onto $q(X)$. The continuum $q(X)$ is chainable. There are four continua $K_{1}, K_{2}, K_{3}, K_{4}$ given by Theorem 1 such that $Z=\cup_{j=1}^{4} K_{j}$ and $f\left(K_{j}\right)=q(X)$ but $f \mid K_{j}$ is not one-to-one, even though $f \mid \operatorname{int}\left(K_{j}\right)$ is one-to-one.

We thank the referee for pointing out that Lemma 2 of this paper appears in a more general form in (4) and that Lemma 1 is also true in a more general form in (7).

\section{REFERENCES}

1. R. H. Bing, Snakelike continua, Duke Math. J., 18 (1951), 653-663.

2. E. Duda and P. Bartick, Finite-to-one Open Mappings on Chainable Continua, Proceedings of Warsaw Topology Conference 1978.

3. E. Duda and J. Kell, Monotone and Open Mappings on Circularly Chainable Continua, Proceedings of Warsaw Topology Conference 1978.

4. E. E. Grace and E. J. Vought, Semi-confluent and weakly confluent images of tree-like and atriodic continua, Fund. Math., 101 (1978), 151-158.

5. W. T. Ingram, Decomposable circle-like continua, Fund. Math., 63 (1968), 193-198.

6. E. S. Thomas, Monotone decompositions of irreducible continua, Rozprawy Matematycne, 50 (1966), 1-73. 
7. E. J. Vought, Monotone decompositions of continua not separated by any subcontinua, TAMS, 192 (1974), 67-78.

8. G. T. Whyburn, Interior transformations on compact sets, Duke Math. J., 3 (1937), 370-381.

Received September 29, 1980 and in revised form December 29, 1981.

UNIVERSITY OF MIAMI

CORAL GABLES, FL 33124 



\section{PACIFIC JOURNAL OF MATHEMATICS EDITORS}

DONALD BABBITT (Managing Editor)

University of California

Los Angeles, CA 90024

Hugo Rossi

University of Utah

Salt Lake City, UT 84112

C. C. Moore and Arthur Ogus

University of California

Berkeley, CA 94720
J. DugunduI

Department of Mathematics

University of Southern California

Los Angeles, CA 90089-1113

R. FinN and H. SAmelson

Stanford University

Stanford, CA 94305

\section{ASSOCIATE EDITORS}
R. ARENS
E. F. BECKENBACH
B. H. NeUmaNN
F. WolF
K. YoshidA (1906-1982)

\section{SUPPORTING INSTITUTIONS}

UNIVERSITY OF ARIZONA

UNIVERSITY OF BRITISH COLUMBIA

CALIFORNIA INSTITUTE OF TECHNOLOGY

UNIVERSITY OF CALIFORNIA

MONTANA STATE UNIVERSITY

UNIVERSITY OF NEVADA, RENO

NEW MEXICO STATE UNIVERSITY

OREGON STATE UNIVERSITY
UNIVERSITY OF OREGON

UNIVERSITY OF SOUTHERN CALIFORNIA

STANFORD UNIVERSITY

UNIVERSITY OF HAWAII

UNIVERSITY OF TOKYO

UNIVERSITY OF UTAH

WASHINGTON STATE UNIVERSITY

UNIVERSITY OF WASHINGTON 


\section{Pacific Journal of Mathematics}

\section{Vol. 106, No. $1 \quad$ November, 1983}

John Ballard, Clifford's theorem for algebraic groups and Lie algebras . . . . . 1

Philip Richard Bartick, II and Edwin Duda, Finite-to-one open mappings

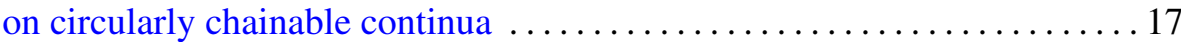

Frank Hayne Beatrous, Jr., $H^{\infty}$-interpolation from a subset of the

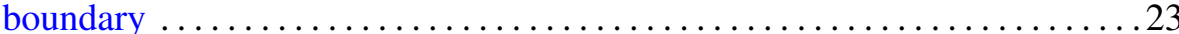

Sterling K. Berberian, Expectations in semifinite algebras ............ 33

Heron S. Collins and Wolfgang Ruess, Weak compactness in spaces of compact operators and of vector-valued functions $\ldots \ldots \ldots \ldots \ldots \ldots . \ldots 45$

David Downing and William O. Ray, Renorming and the theory of

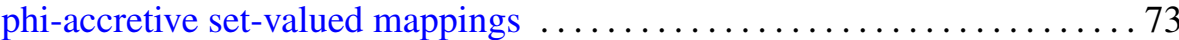

Harvey Charles Greenwald, On the theory of homogeneous Lipschitz

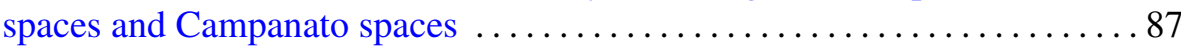

Irwen Valle Guadalupe and Lucio Ladislao Rodriguez, Normal curvature

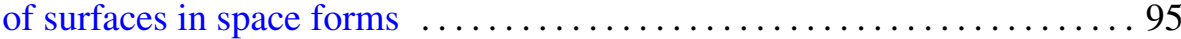

Boju Jiang, On the computations of the Nielsen number ............... 105

William H. Julian, Ray Mines, III and Fred Richman, Alexander

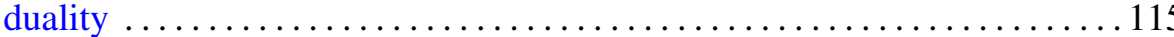

Midori Kobayashi, The connected component of the idèle class group of an

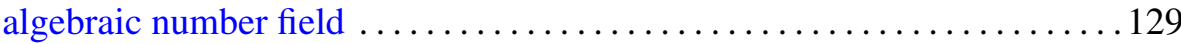

Ib Henning Madsen, Charles B. Thomas and C. Terence C. (Charles)

(Clegg) Wall, Topological spherical space form problem. III.

Dimensional bounds and smoothing

A. Sapounakis, The existence of strong liftings for totally ordered measure

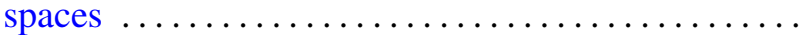

Gary Seitz, The root subgroups for maximal tori in finite groups of Lie type

Laurent Siebenmann and James M. Van Buskirk, Construction of irreducible homology 3-spheres with orientation reversing involution 